\title{
Minimal Simplicial Dissections and Triangulations of Convex 3-Polytopes*
}

\author{
A. Below, ${ }^{1}$ U. Brehm, ${ }^{2}$ J. A. De Loera, ${ }^{3}$ and J. Richter-Gebert ${ }^{1}$ \\ ${ }^{1}$ Institut für Theoretische Informatik, ETH-Zürich, \\ CH-8092 Zürich, Switzerland \\ \{below.richter\}@inf.ethz.ch \\ ${ }^{2}$ Institut für Geometrie, Technische Universität Dresden, \\ 01062 Dresden, Germany \\ brehm@math.tu-dresden.de \\ ${ }^{3}$ Department of Mathematics, University of California at Davis, \\ Davis, CA 95616-8633, USA \\ deloera@math.ucdavis.edu
}

\begin{abstract}
This paper addresses three questions related to minimal triangulations of a three-dimensional convex polytope $P$.

- Can the minimal number of tetrahedra in a triangulation be decreased if one allows the use of interior points of $P$ as vertices?

- Can a dissection of $P$ use fewer tetrahedra than a triangulation?

- Does the size of a minimal triangulation depend on the geometric realization of $P$ ?
\end{abstract}

The main result of this paper is that all these questions have an affirmative answer. Even stronger, the gaps of size produced by allowing interior vertices or by using dissections may be linear in the number of points.

\section{Introduction}

Let $\mathcal{A}$ be a point configuration in $\mathbb{R}^{d}$ with its convex hull $\operatorname{conv}(\mathcal{A})$ having dimension $d$. A set of $d$-simplices with vertices in $\mathcal{A}$ is a dissection of $\mathcal{A}$ if no pair of simplices has a common interior point and their union equals $\operatorname{conv}(\mathcal{A})$. A dissection is a triangulation

\footnotetext{
* Most of the results of this paper had been obtained and presented [6] by the second author around 1992, but remained unpublished. The remaining authors solved these problems independently after several researchers brought them to their attention. Here we present a joint final version.
} 
of $\mathcal{A}$ if in addition any pair of simplices intersects in a common face. Following the standard terminology in the literature (see, for instance, [15]), we say that a triangulation (dissection) of a convex polytope $P$ is a triangulation (dissection) of the vertices of $P$. Notice that in this definition no additional points are allowed. In contrast to this, we are interested in triangulations of point sets with additional interior points besides the vertices of $P$ and call them triangulations of $P$ with interior points. The size of a dissection $D$ is the number of $d$-simplices it contains and we denote it by \#D. We call a triangulation or a dissection minimal/maximal when its size is the smallest/largest among all triangulations or respectively all dissections. Note that dissections form simplicial complexes precisely if they are triangulations.

In this paper we solve three questions about triangulations and dissections, and present several relevant consequences.

In Section 3 we investigate how the size of a minimal triangulation changes under the addition of new interior points. Independently Böhm [5] and Gritzmann and Klee [11] raised the issue that possibly using auxiliary interior points the size of a minimal triangulation of the new set of points could be smaller than a minimal triangulation of the original set. As pointed out in Section 8.4 of [11], this question is relevant in the study of complexity classes of basic problems in computational convexity. In this article we exhibit a family of three-dimensional polytopes that indeed have this property. This behavior is reminiscent of the fact that adding points can also reduce the size of a Delaunay triangulation [3], [7].

We show in Section 3 that dissections can require fewer simplices than triangulations. We show that a linear difference between the size of a minimal triangulation and a minimal dissection is possible. The question whether this could happen had been raised several times [5], [8], [13], [11]. The solution of the questions depends on two simple geometric lemmas that will be useful throughout the paper. They are proved in Section 2. The main results of Section 3 can be stated as follows:

Theorem 1.1. There is a family of simplicial convex 3-polytopes $P_{m}$ with the following properties:

(1) All triangulations of $P_{m}$ are larger than a triangulation with one interior point. This gap can be linear in the number of vertices.

(2) All triangulations of $P_{m}$ are larger than the minimal dissections of $P_{m}$. This gap can be linear in the number of vertices.

It should be pointed out that after the proof of Theorem 1.1 we construct a "twisted" version of $P_{m}, P_{m, \alpha}$, for which it is clear to see (with essentially the same proof) that dissections are also smaller than triangulations of $P_{m, \alpha}$ with interior points.

Moreover, we show that the approximate shape of the polytope can be prescribed and still have interesting behavior:

\section{Theorem 1.2.}

(1) Given three numbers $h_{0} \geq 1, k \geq 1, \varepsilon>0$ and a convex body $K \subseteq \mathbb{R}^{3}$ there is a simplicial convex 3-polytope $P$ with $\delta(K, P)<\varepsilon$ (Hausdorff distance) such that every triangulation of $P$, with up to $h_{0}-1$ interior points, has at least $k$ 
tetrahedra more than a triangulation of $P$ with $h_{0}$ suitably chosen additional interior points.

(2) For a 3-polytope $P$, if adding $n^{\prime}$ new interior points allows for a smaller triangulation than a minimal triangulation of $P$, then $n^{\prime}<e_{i}$ where $e_{i}$ is the number of interior edges in a minimal triangulation of $P$. In particular, it is impossible to have such behavior for 3-polytopes whose minimal triangulations use one or no interior edges.

A famous open question in computational geometry asks for the computational complexity of finding minimal triangulations of convex polyhedra [2]. A related problem is whether the smallest size of a triangulation of a convex polytope is determined by the face lattice or if it can change with the coordinates. We present the negative answer in Section 4:

Theorem 1.3. The minimal size of a triangulation of a convex 3-polytope is not an invariant of the face lattice. There is a simplicial convex 3-polytope with 10 vertices for which the minimal number of tetrahedra possible in a triangulation depends on its coordinates. The example is smallest possible in dimension and number of vertices.

The theory of universal polytopes [4], [9] implies that if two polytopes have the same oriented matroid generated by their convex hull vertices, then not only are their face lattices the same but also their set of triangulations (without interior points) are identical. In contrast to this Brehm has proved that in fact, if additional interior points are allowed the minimal number of tetrahedra of a dissection or triangulation is not an invariant of the oriented matroid of the vertices in the convex hull. Details on this result will appear elsewhere.

\section{Two Useful Lemmas}

The following elementary formula shows that many of the arguments about the number of tetrahedra in a triangulation can be reduced to the study of the number of interior edges. Similar formulas have already been stated many times in the literature (see, for instance, Lemma 2.1 in [10] or [17]). The proof is a simple application of Euler's formula for triangulated 2-spheres and 3-balls and we omit the easy details.

Lemma 2.1. Let $P$ be a 3-polytope with $n$ boundary vertices and $n^{\prime}$ interior auxiliary vertices. For a triangulation $T$ of $P$ that uses the $n^{\prime}$ interior points, the number of tetrahedra in $T$ is related to the number of interior edges $e_{i}$ of $T$ by the formula

$$
\# T=e_{i}+n-3-n^{\prime} .
$$

We now present a lemma that showcases the main nontrivial effect we use in all the examples. The lemma shows that a certain substructure in the face lattice of a polytope forces certain interior edges to appear in triangulations of sufficiently small size. 


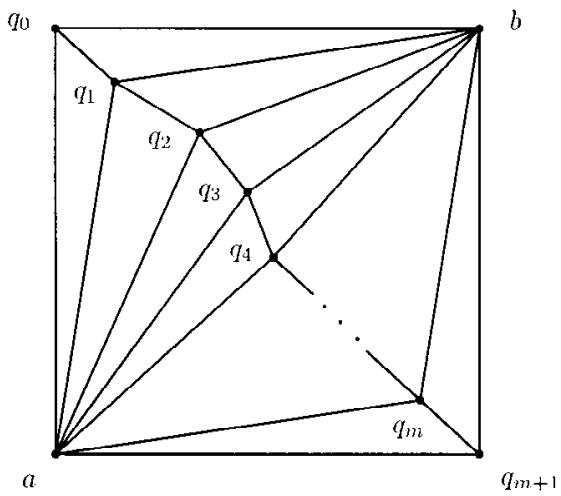

Fig. 1. The key structure of Lemma 2.2.

Lemma 2.2. Let $P$ be a convex 3-polytope on $n$ vertices, that contains the following collection of triangularfacets: $\left[a, q_{i}, q_{i+1}\right]$ and $\left[b, q_{i}, q_{i+1}\right]$ for $i=0, \ldots, m$ (see Fig. 1), with the additional restrictions that conv $\{a, b\}$ does not intersect conv $\left\{q_{0}, \ldots, q_{m+1}\right\}$. Then for each triangulation of $P$ that does not use the edge $(a, b)$ the number of interior edges $e_{i}$ is at least $m$.

Proof. Since $\operatorname{conv}\left\{q_{0}, q_{m+1}\right\}$ is in the interior of $P$, we obtain the following simple fact: for all $|i-j| \geq 2$, if $q_{i} q_{j}$ is an edge of a triangulation, it will also be an interior edge. The proof of the lemma proceeds by induction on $m$. The lemma is clearly true for $m=1$. Call (*) the assumption that all vertices $q_{i}$, with $1 \leq i \leq m$, are incident to at least one interior edge of the triangulation $T$. We now show how to invoke induction in case (*) does not hold: A vertex $q_{i}$ untouched by an interior edge belongs to the tetrahedra $\sigma_{i, a}=\left(a, q_{i-1}, q_{i}, q_{i+1}\right)$ and $\sigma_{i, b}=\left(b, q_{i-1}, q_{i}, q_{i+1}\right)$. This is because the triangle $\left(a, q_{i}, q_{i+1}\right)$ is in some simplex, and if the fourth point is some other vertex besides $q_{i-1}$ or $b$ we have an interior edge touching $q_{i}$. Furthermore, the fourth point cannot be $b$ since in this case the edge $a b$ would be present. By chopping off these two tetrahedra together with the vertex $q_{i}$ (i.e., considering the convex hull of all of $P$ 's vertices except $q_{i}$ ) we can apply induction to guarantee that the remaining triangulation $T \backslash \sigma_{i, a}, \sigma_{i, b}$ has at least $m-1$ interior edges. Together with the edge $q_{i-1} q_{i+1}$ they account for $m$ interior edges in $T$.

If $\left(^{*}\right)$ holds we will show the claim directly; we set up a one-to-one map from the set $\left\{q_{1}, \ldots, q_{m}\right\}$ to a subset of the interior edges that touch them: The vertices $q_{i}$ come along a polygonal curve in a canonical order which is reflected by their indices. We mark or orient the interior edges $q_{i} v$ that touch a vertex $q_{i}$ as follows: If $v \notin\left\{q_{0}, \ldots, q_{m+1}\right\}$, we call the edge $q_{i} v$ special, otherwise we orient it from smaller to larger index. For the vertices $q_{i}$ with special edges incident to them, we map $q_{i}$ to one of those. If a vertex $q_{i}$ has no special edges, but has outgoing interior edges, we map it to the outgoing edge $q_{i} q_{k}$ with the smallest index $k$. We are left with the case of those vertices $q_{i}$ that have only incoming interior edges incident to $q_{i}$. Consider the triangle $\left(a, q_{i}, q_{i+1}\right)$. It has to be in some tetrahedron of $T$ whose fourth point is bound to be a $q_{j_{a}}$ with $j_{a}<i$. Likewise 
$\left(b, q_{i}, q_{i+1}\right)$ is in a tetrahedron with fourth point $q_{j_{b}}$ with $j_{b}<i$. If both $j_{a}=j_{b}=i-1$, there can be no interior edges incident to $q_{i}$ (see above), a contradiction to (*). Let $j$ be any of $j_{a}, j_{b}$ such that $j<i-1$. Map $q_{i}$ to $q_{j} q_{i+1}$.

We claim that the given map is one-to-one. If some vertex $q_{i}$ maps to the special edge $q_{j} v$, then necessarily $i=j$. There are potentially two vertices that can be mapped to an interior edge $q_{j} q_{k}$ with $j<k: q_{j}$ when $q_{j} q_{k}$ is the chosen outgoing edge of $q_{j}$ and $q_{k-1}$, in case $q_{k-1}$ has only incoming edges. In the latter case one of the tetrahedra $\left(a, q_{j}, q_{k-1}, q_{k}\right)$ and $\left(b, q_{j}, q_{k-1}, q_{k}\right)$ has to be in the triangulation, and $q_{j}$ will be mapped to the smaller indexed edge $q_{j} q_{k-1}$. This is an interior edge since $j<k-2$, so $q_{j}$ cannot also be mapped to $q_{j} q_{k}$. The injectivity of the map is proven.

Roughly speaking the purpose of Lemma 2.2 is the possibility to force the occurrence of many interior edges when other interior edges are absent. Our solutions to the open questions rely on a combination of this effect with a famous example, Schönhardt's example of a nontriangulable nonconvex polyhedron (see [15]-[16], [18], and [19]). The example, obtained from "twisting" a triangular prism and allowing that the three diagonals bend in, has the property that it cannot be triangulated or dissected unless we add a new point and then the triangulation is unique. A variation of the same example is a triangulation of the boundary of a triangular prism that chooses boundary edges in a "cyclic" manner. Such triangulation of the boundary cannot be extended to a triangulation of the convex hull.

\section{Adding Points or Dissecting Can Reduce the Size}

Proof of Theorem 1.1. Consider a triangular prism with bottom triangle $\left(A_{1}, A_{2}, A_{3}\right)$ and top triangle $\left(B_{1}, B_{2}, B_{3}\right)$ where both triangles are congruent, parallel, equilateral, and vertex $A_{i}$ lies directly below vertex $B_{i}$. Denote by $n_{i, j}$ the inner normal to the quadrilateral facet $\left(A_{i}, A_{j}, B_{i}, B_{j}\right)$. In each of its quadrilateral facets we will put $m$ points following a diagonal $A_{i} B_{j}$ but slightly lifted to be in a parabola. The points we add will have coordinates along three parabolic curves $C_{1,2}, C_{2,3}$, and $C_{3,1}$. Let $p(\lambda)=\left(\lambda-\frac{1}{2}\right)^{2}-\frac{1}{4}$, and $\varepsilon>0$ a sufficiently small number, we define

$$
\begin{aligned}
& C_{1,2}=\lambda A_{1}+(1-\lambda) B_{2}+\varepsilon p(\lambda) n_{1,2}, \\
& C_{2,3}=\lambda A_{2}+(1-\lambda) B_{3}+\varepsilon p(\lambda) n_{2,3}, \\
& C_{3,1}=\lambda A_{3}+(1-\lambda) B_{1}+\varepsilon p(\lambda) n_{3,1} .
\end{aligned}
$$

Note that the curve $C_{i, j}$ joins the vertices $A_{i}$ and $B_{j}$. The pattern of the curves follows cyclically arranged diagonals as in the case of Schönhardt's polytope. Take $m$ points along each of the curves $C_{1,2}, C_{2,3}, C_{3,1}$ that we denote by $q_{1}\left(C_{i, j}\right), q_{2}\left(C_{i, j}\right), \ldots, q_{m}\left(C_{i, j}\right)$. We will take those points such that all the triangles $\left(q_{k}\left(C_{1,2}\right), B_{2}, B_{3}\right)$ are pierced by the edge $B_{1} A_{2}$, the triangles $\left(q_{k}\left(C_{2,3}\right), B_{3}, B_{1}\right)$ are pierced by the edge $B_{2} A_{3}$, and similarly the triangles $\left(q_{k}\left(C_{3,1}\right), B_{1}, B_{2}\right)$ are pierced by the edge $B_{3} A_{1}$. These piercing conditions are easily achieved by accumulating the points low enough toward the triangle $\left(A_{1}, A_{2}, A_{3}\right)$. We observe that each of the sequences of points produces, when taking the convex hull, $2(m+1)$ triangular facets, $m+1$ on each side of the prism. This is exactly the situation 
$B_{1}$

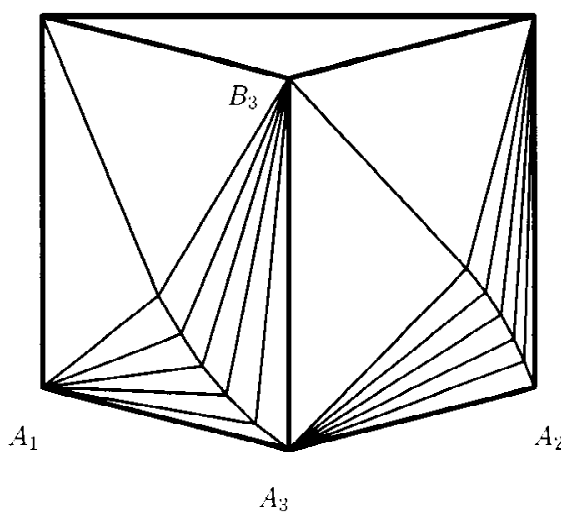

$B_{2}$

$A_{2}$

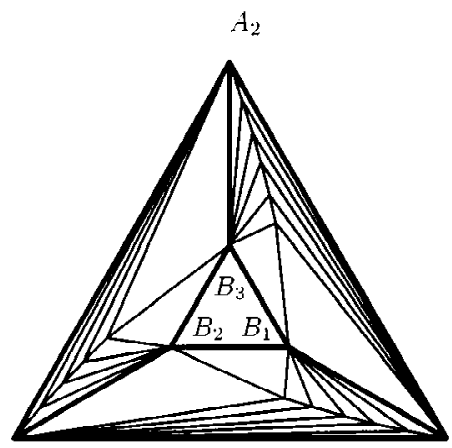

$A_{3}$

$A_{1}$

Fig. 2. The polytope $P_{5}$ and its Schlegel diagram.

of Lemma 2.2. The resulting 3-polytope, containing $3 m+6$ vertices, will be denoted by $P_{m}$. The polytope $P_{m}$ is the union of the original prism with three "caps" placed on top of the quadrilateral facets. We show in Fig. 2 the case for $m=5$.

Now we construct a triangulation $T_{v}$ of $P_{m}$ that uses an auxiliary interior point $v$ within the prism $\operatorname{conv}\left(A_{1}, A_{2}, A_{3}, B_{1}, B_{2}, B_{3}\right)$. At each cap of the polytope $P_{m}$ we triangulate in a "stacked" way, namely, by choosing the tetrahedra of the form $\left(q_{k}\left(C_{i, j}\right), q_{k+1}\left(C_{i, j}\right)\right.$, $\left.B_{i}, A_{j}\right)$. For each curve $C_{i, j}$ we get $m+1$ tetrahedra. Complete the triangulation by coning from $v$ to the eight triangles that triangulate the boundary of $\operatorname{conv}\left(A_{1}, A_{2}, A_{3}, B_{1}, B_{2}, B_{3}\right)$. We have a triangulation, using the interior point $v$, with $3 m+11$ tetrahedra.

We now construct a dissection $D$ of $P_{m}$. We do not use any interior points. Triangulate the three caps as before in a stacked way, then for the interior of the prism triangulate arbitrarily. All triangulations of the prism are combinatorially the same and have three simplices. The dissection $D$ has size $3 m+6$.

From Lemma 2.2 we have that triangulations that do not use all the edges $B_{1} A_{2}$, $B_{2} A_{3}$, or $B_{3} A_{1}$ will have at least $m$ interior edges. In this case Lemma 2.1 implies that the number of tetrahedra will be at least $4 m+3$. On the other hand, we claim that it is impossible to have a triangulation that uses those three edges simultaneously. The reason is that the triangular face $\left(B_{1}, B_{2}, B_{3}\right)$ must belong to a certain tetrahedron, and if the fourth point is along one of the curves $C_{i, j}$, then, by the piercing conditions, there is a bad intersection with one of the edges $B_{1} A_{2}, B_{2} A_{3}$, or $B_{3} A_{1}$. Hence, the only hope is that the fourth point is another of the points $A_{l}$, but this is impossible in a triangulation as two edges will intersect improperly. In conclusion, any triangulation of $P_{m}$ that does not use interior points is larger than the triangulation $T_{v}$ and larger than the dissection $D$ for $m>8$.

Before we come to the proof of Theorem 1.2, we present some observations on a twisted version of polytope $P_{m}$. We will patch several copies of this new polytope onto simplicial polytopes when constructing the examples of Theorem 1.2. We start with a triangular prism such that the bottom triangle $\left(A_{1}, A_{2}, A_{3}\right)$ and the top triangle 

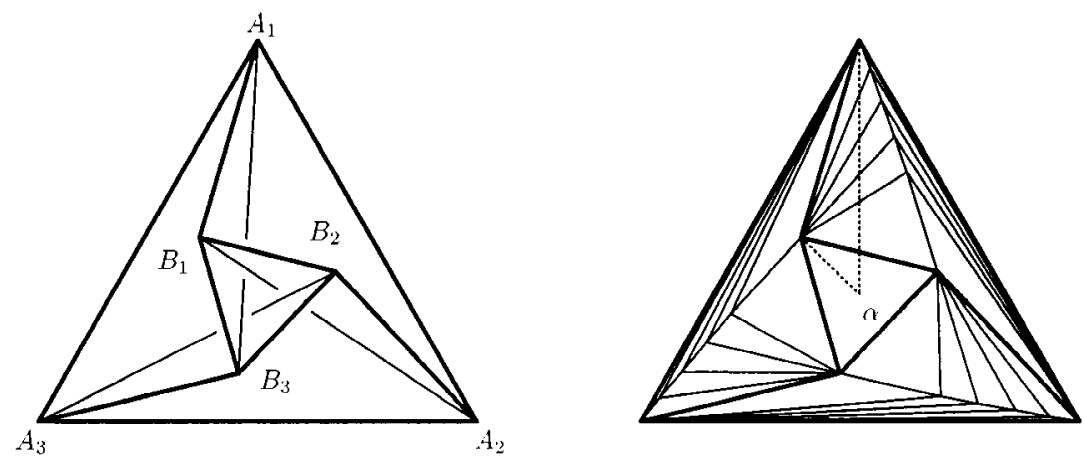

Fig. 3. A twisted version of $P_{m}$, based on Schönhardt's example.

$\left(B_{1}, B_{2}, B_{3}\right)$ are equilateral, and their barycenters are vertically on top of each other. The bottom triangle should be somewhat larger than the top triangle. We twist the top triangle by an angle of $\alpha$ degrees creating a Schönhardt style nonconvex polyhedron. Then we patch the sides again with copies of the caps introduced in Lemma 2.2. We call the resulting convex polytope $P_{m, \alpha}$. Therefore the original example $P_{m}$ of Theorem 1.1 is isomorphic to $P_{m, 0}$ (see Fig. 3). The copies will again have the points $q_{k}\left(C_{i, j}\right)$ close to the bottom triangle to guarantee the piercing conditions as in the proof of Theorem 1.1. The number $\varepsilon$ should be small enough for the points $q_{k}\left(C_{i, j}\right)$ to lie vertically above the bottom triangle. By vertically above (below) we mean that a line going vertically down (up) from any of those points pierces $\left(A_{1}, A_{2}, A_{3}\right)$.

When the angle $\alpha$ is greater than zero, we are in the situation that the dissection $D$ we described becomes impossible, but one can still use an interior point $v$ to create a triangulation $T_{v}$ with an interior point, smaller than any triangulation of the polytope. The proof is identical to the one we discussed for Theorem 1.1.

An important difference from the example of Theorem 1.1 is that now the set of possible interior points $v$ to construct $T_{v}$ is restricted by the twisting angle $\alpha$. We call the set of visibility of $P_{m, \alpha}$ all points $p$ which are below the plane spanned by the top triangle and satisfy the following condition: the tetrahedron spanned by $B_{1}, B_{2}, B_{3}$, and $p$ does not intersect the edges $B_{1} A_{2}, B_{2} A_{3}$, or $B_{3} A_{1}$. It is easy to check that the set of visibility is the union of the following four sets (see Fig. 3): the tetrahedron $S_{0}$ bounded by the planes $B_{1} B_{2} B_{3}, B_{1} A_{1} B_{3}, B_{2} A_{2} B_{1}$, and $B_{3} A_{3} B_{2}$; the cone $S_{1}$, with apex $B_{1}$ lying between the planes $B_{1} B_{2} B_{3}, B_{1} A_{1} B_{3}$, and $B_{1} A_{3} B_{2}$; as well as the analogous cones $S_{2}$ and $S_{3}$ with apexes $B_{2}$ and $B_{3}$. The reason we want the piercing conditions is exactly to avoid this set of visibility.

By symmetry, the three planes $B_{1} A_{1} B_{3}, B_{2} A_{2} B_{1}$, and $B_{3} A_{3} B_{2}$ intersect in a point $p$ which lies on the vertical line connecting the barycenters of the bottom and top faces. If the twisting angle $\alpha=\pi / 3$, then $p$ is the intersection of edges $B_{1} A_{2}, B_{2} A_{3}$, and $B_{3} A_{1}$. By continuity of the rotation, if $\alpha=\pi / 3-\delta$ for small $\delta>0$, the point $p$ is still above the bottom triangle. Therefore $S_{0}$ has no points vertically below $\left(A_{1}, A_{2}, A_{3}\right)$. Note that the sets $S_{1}, S_{2}, S_{3}$ do not contain points vertically below the bottom triangle. 
In conclusion, for a suitable twisting angle $\alpha$ the polytope $P_{m, \alpha}$ will have four properties: (1) it contains only points in and vertically above its bottom face, (2) there are no points of visibility vertically below the bottom triangle, (3) inside $P_{m, \alpha}$ there is a point which can "see" the top and (by an analogous argument) the bottom triangular faces, and (4) a new auxiliary interior point $v$ used in a smaller triangulation $T_{v}$ must lie strictly in the set of visibility of $P_{m, \alpha}$.

Imagine now a polytope $Q$, with facet $\left(A_{1}, A_{2}, A_{3}\right)$, lying vertically below this facet. Then $Q \cup P_{m, \alpha}$ is a convex polytope. There is a triangulation of $Q \cup P_{m, \alpha}$ using an interior point in $P_{m, \alpha}$ which does not use $A_{1} B_{3}, A_{2} B_{1}, A_{3} B_{2}$, but there is no such triangulation if we do not allow extra interior points within $P_{m, \alpha}$. Via a projective transformation we can transform any polytope $Q^{\prime}$ with a specified triangular facet $\left(A_{1}^{\prime}, A_{2}^{\prime}, A_{3}^{\prime}\right)$ to such a polytope $Q$. We can even arrange for the preimage of $P_{m, \alpha}$ under this projective transformation to be as "flat" as we want it to be. Hence we can patch arbitrarily flat isomorphic copies of $P_{m, \alpha}$ to any triangular facet of $Q^{\prime}$ still obtaining a convex polytope.

Proof of Theorem 1.2(1). Approximate the convex body $K$ with a simplicial 3-polytope $Q_{0}$, with at least $h_{0}$ facets, that is close to it in the Hausdorff distance (see [12] for references on approximation by polytopes). On $h_{0}$ of the triangular facets of $Q_{0}$ we stack polytopes $Y_{1}, Y_{2}, \ldots, Y_{h_{0}}$ where each $Y_{i}$ is an isomorphic copy of a polytope $P_{m, \alpha}$ and $Q_{1}=Q_{0} \cup Y_{1} \cup Y_{2} \cup \cdots \cup Y_{h_{0}}$ is a convex polytope still $\varepsilon$-close to $K$. This stacking procedure is possible by the above discussion.

If one triangulates $Y_{i}$ with an interior point that lies in its visibility set one finds a very small triangulation, but the auxiliary point is forced to lie in the visibility sets by construction of each copy $Y_{i}$. Moreover, the visibility sets of different $Y_{i}, Y_{j}$ are disjoint from each other and from $Q_{0}$. The rest of $Q_{1}$ is the original polytope $Q_{0}$ which we triangulate minimally.

A simple calculation shows that in the case where we use all $h_{0}$ interior points the triangulation can be done with at most $C\left(3 m+C^{\prime}\right)$ tetrahedra. Otherwise, if we do not use all $h_{0}$ additional interior points, any triangulation of the polytope $Q_{1}$ requires at least $m$ additional tetrahedra (in the above $C, C^{\prime}$ are constants). Thus if we add $h_{0}$ points to the interior of $Q_{1}$ we can produce a triangulation that has at least $k$ fewer tetrahedra since for large enough $m$ we can surpass any value $k$ we are given.

Proof Theorem 1.2(2). We use the equation \#T $=e_{i}+n-3-n^{\prime}$ from Lemma 2.1 where \#T denotes the number of tetrahedra in $T, n\left(n^{\prime}\right)$ equals the number of boundary (interior) vertices, and $n^{\prime}=0$ before we add interior points.

If using the $n^{\prime}$ interior auxiliary points reduces the size of the triangulation, then we get the inequality $e_{i}^{*}+n-3-n^{\prime}<e_{i}+n-3$, where $e_{i}^{*}$ is the number of interior edges in the new triangulation $T^{\prime}$ that uses interior vertices. Notice that since every interior point is in at least four interior edges, $4 n^{\prime} \leq 2 e_{i}^{*}$. In conclusion $n^{\prime}<e_{i}$. The rest of the statement follows immediately.

Note that if in the triangulation $T_{v}$ of Theorem 1.1 we take the new point $v$ not in the interior of $P_{m}$, but rather on the relative interior of $\left(A_{1}, A_{2}, A_{3}\right)$, then there is 
an even bigger difference between the size of $T_{v}$ and any triangulation of $P_{m}$ because there are at least three fewer interior edges. Hence we conclude that triangulating a convex polytope using new auxiliary boundary points can result in smaller triangulations as well. Similarly, adding exterior points, placed beyond a facet, could also help to reduce the size of a minimal triangulation (place the new point point beyond $\left.\left(A_{1}, A_{2}, A_{3}\right)\right)$.

Remarks. (1) In his investigations Brehm introduced two interesting notions [6]: An $f$-simplicial dissection of a polytope $P$ is a collection of simplices that forms a simplicial complex (every pair of simplices intersects in a common face, possibly empty), the union of the simplices equals $P$, but at the same time some of the simplices may be "degenerate." Degenerate means that their $d+1$ vertices may not be affinely independent. For example, a regular octahedron has an $f$-simplicial dissection with five tetrahedra, one of them degenerate. The other notion is the excess. The excess of a triangulation or dissection $D$ of a polytope $P$ is defined as \#D\#vertices $(P)+3$. In this way, the excess is zero for a minimal triangulation of a stacked polytope and from Lemma 2.1 it coincides with the number of interior edges minus the number of interior vertices in the triangulation. The excess can be thought of as a measure of how simple a dissection, triangulation, or $f$-simplicial dissection can be. It is interesting to see how the excess of a dissection, triangulation, or $f$ simplicial dissection changes (is reduced) by adding points in the interior or the boundary. One can observe also changes in the excess between the example $P_{m}$ and its twisted version

(2) Our paper suggests a new definition of minimal triangulation of a convex polytope. Rather than prescribing the vertices in advance, one can define it as an intrinsic property of the convex body itself allowing the possibility of new interior points. We did not take this point of view in the statements of results because it is traditional in the literature of triangulations of polytopes to fix the point set in advance (in this way the set of all possible triangulations is a finite set).

\section{Minimal Triangulations and Coordinate Changes}

A natural question is whether the size of a minimal triangulation could be an invariant of the face lattice. We present the smallest example of a polytope where this invariance fails to exist. This implies that any algorithm to compute minimal triangulations of 3 -polytopes must take into account the coordinates of the polytope, not only its face lattice.

Proof of Theorem 1.3. The example is given by the 3-polytope with 10 vertices shown in Fig. 4. The polytope is made by superimposing two copies of the combinatorial structure used in Lemma 2.2. If the points $A, B, C, D$ are coplanar, then one of the edges $A B$ or $C D$ cannot be in a triangulation at the same time. Hence by Lemmas 2.1 and 2.2, any triangulation has at least 10 simplices.

On the other hand, one can arrange the $z$-coordinates of $A, B, C$, and $D$ in such a 


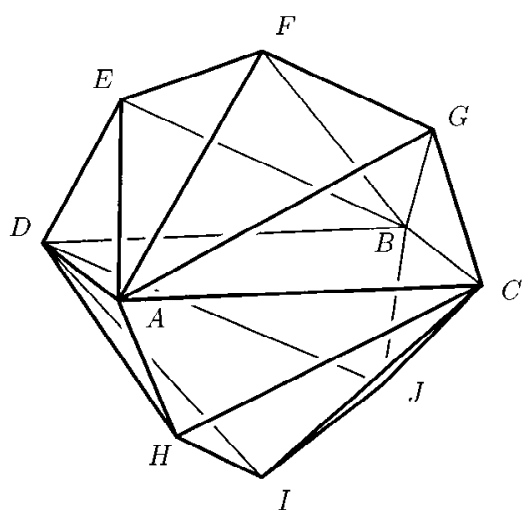

Fig. 4. A view of the polyhedron with variable minimal triangulation.

way that the edge $A B$ is above the edge $C D$. This way it has a unique minimal triangulation with nine simplices: $(A, C, D, H),(A, B, C, D),(A, B, E, F),(A, B, D, E)$, $(C, D, I, J),(A, B, C, G),(C, D, H, I),(A, B, F, G),(B, C, D, J)$. The triangulation above is possible since the tetrahedron $(A, B, C, D)$ is nondegenerate and does not intersect the other simplices.

The fact that this example is minimal in dimension and number of vertices is a consequence of the following lemmas:

Lemma 4.1. If a triangulation $T$ of a 3-polytope $P$ uses at most one interior edge, then for any polytope $P^{\prime}$ with the same face lattice, the set of simplices $T^{\prime}$ corresponding to $T$ is still a triangulation.

Proof. We have to show two things: First, that $T^{\prime}$ still defines a simplicial complex, i.e., the tetrahedra of $T^{\prime}$ are all full-dimensional and intersect properly. Second, that all points of $P^{\prime}$ belong to one of the tetrahedra of $T^{\prime}$.

Changing the coordinatization of $P$ to the coordinates of $P^{\prime}$ while maintaining the face lattice gives us a bijection $f$ of the vertices of $P$ and of $P^{\prime}$. In our proof we refer to the vertices of $P^{\prime}$ by primed letters, e.g., $f(v)=v^{\prime}$ for $v$ a vertex of $P$. The bijection $f$ can be canonically extended to map $T$ to $T^{\prime}$, or, for that matter, (abstract) faces of simplices in $T$ to (abstract) faces of simplices in $T^{\prime}$. Note that interior edges in $P$ are always mapped to interior edges in $P^{\prime}$, and that edges on the boundary will stay on the boundary. For the proof of the lemma we use that the vertices in $P^{\prime}$ lie in convex position and that the simplices in $T^{\prime}$ use at most one interior edge.

We prove now that the tetrahedra of $T^{\prime}$ are all full-dimensional. Any simplex in $T^{\prime}$ uses at most one interior edge. It has therefore vertices $v_{1}, v_{2}$, and $v_{3}$ which are connected by edges which lie on the boundary. If the fourth point had collapsed into a coplanarity with the other three points, it is forced, by $v_{1} v_{2}, v_{2} v_{3}, v_{1} v_{3}$ being boundary edges, to lie in $\operatorname{conv}\left(v_{1}, v_{2}, v_{3}\right)$. This contradicts its being an extreme point in the convex hull. Hence all simplices of $T^{\prime}$ are full-dimensional. 


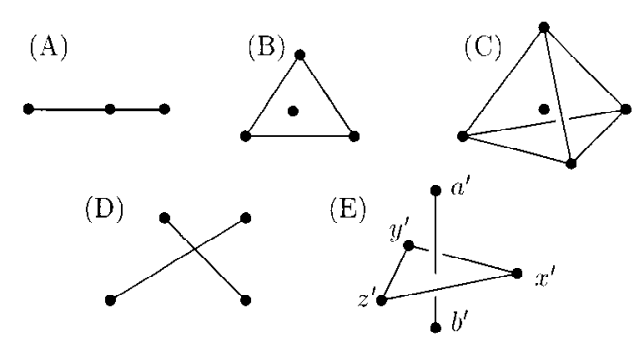

Fig. 5. Possible bad intersections of faces of tetrahedra.

Suppose two simplices in $T^{\prime}$ intersect improperly; that is, not in a common face. Such a bad intersection occurs if a pair of faces of $T^{\prime}$ forms a new minimal Radon partition (their relative interiors intersect) or in oriented matroid terminology a circuit [21]. In three dimensions the only five possible circuits are presented in Fig. 5. Obviously, the cases (A), (B), and (C) change the face lattice when they occur because a vertex becomes an interior point.

Consider now the pair of edges related to case (D). At least one of them is exterior since we only have one interior edge inside $T$. Their intersection point is therefore on the boundary of $P^{\prime}$. Hence both edges have to be exterior edges lying in a common two-face of $P^{\prime}$. This implies that their preimages in $P$ already intersected improperly which is impossible since $T$ was a triangulation. Case (D) is forbidden.

For case (E) note that if the edge $a^{\prime} b^{\prime}$ intersects the triangle $\left(x^{\prime} y^{\prime} z^{\prime}\right)$ in $P^{\prime}$, then $a^{\prime} b^{\prime}$ is interior; leaving the other three edges $x^{\prime} y^{\prime}, x^{\prime} z^{\prime}, y^{\prime} z^{\prime}$ to be exterior. This is also true for their preimages. This forces that, for the old coordinates when relint $(a b)$ and relint $(x y z)$ were disjoint, the vertices $a, b$ were in the same side of the hyperplane spanned by $x, y$, and $z$; otherwise one of the edges $x y, x z, y z$ is also interior. Finally, the vertices $x, y, z$ form a cut of the graph of the polytope because their edges are all in the boundary. However, then the face lattice was changed when going from the old to the new coordinates because the cut leaves the points $a, b$ on the same side of the hyperplane but later they lie on opposite sides.

We have shown that the simplices in $T^{\prime}$ form a simplicial complex. Finally, we show that every point of $P^{\prime}$ belongs to one simplex of $T^{\prime}$. Denote by $\left|T^{\prime}\right|$ the point set $\bigcup_{\sigma^{\prime} \in T^{\prime}} \sigma$ (similarly for $|T|$ ). We can assume the bijection $f$ is extended to a one-to-one point map $f:|T|=P \mapsto\left|T^{\prime}\right|$, and it can be made continuous, e.g., by taking the canonical affine map on each simplex given by the values of the vertices of that simplex. Note that the boundary of $P$ is mapped bijectively to the boundary of $P^{\prime}$. If there were points in $P^{\prime}$ which are not any simplex of $T^{\prime}$, they have to be in the interior of $P^{\prime}$. Hence, the set of all those points equals $\operatorname{int}\left(P^{\prime}\right) \backslash\left|T^{\prime}\right|$ which is an open set. Actually, so is $\operatorname{int}\left(P^{\prime}\right) \cap\left|T^{\prime}\right|$ since the boundary of $\left|T^{\prime}\right|$ is by continuity and bijectivity of $f$ the boundary of $P^{\prime}$. This would imply having a partition of $\operatorname{int}\left(P^{\prime}\right)$ into two open sets which contradicts the connectivity of $\operatorname{int}\left(P^{\prime}\right)$ in a topological sense. This concludes the proof of the lemma.

Lemma 4.2. For convex 3-polytopes with up to nine vertices, the smallest possible number of tetrahedra in a triangulation is determined by the face lattice. 
Proof. First we give necessary bounds for the number of vertices $n$ and number of tetrahedra $t$ in a minimal triangulation that is not present in every coordinatization.

From Lemma 2.1 we know that a triangulation of a polytope with fewer than $n-1$ tetrahedra has at most one interior edge. By the previous lemma, those triangulations are invariant under coordinate changes, hence $t \geq n-1$.

A triangulation that exists for all coordinatizations can be made as follows: select a vertex $v$, triangulate all facets of $P$ that do not contain $v$. The tetrahedra of the triangulation of $P$ are those formed by $v$ and a triangle in the boundary not in a facet containing $v$. This procedure is called coning from vertex $v$ or triangulating by pulling, see [15] or [4] and [14]. The number of tetrahedra in this triangulation is the number of triangles in the triangulated boundary minus the number of triangles in the faces that touch $v$. The first number is $2 n-4$ by Euler's formula. The second number is the degree of $v$ in the graph $G_{\text {face }}$ consisting of the vertices of $P$ and all edges between vertices lying in a common face. This graph contains the graph $G^{\prime}$ of any triangulation of the boundary. By Euler's formula, $G^{\prime}$ has $3 n-6$ edges, hence the maximal degree in $G^{\prime}$ (and therefore in $G_{\text {face }}$ ) is at least $\lceil 2 \cdot((3 n-6) / n)\rceil$.

We showed that pulling from a vertex $v$ with maximal degree in $G_{\text {face }}$ gives an invariant triangulation with at most $s(n):=2 n-4-\lceil 2 \cdot((3 n-6) / n)\rceil$ tetrahedra. A noninvariant minimal triangulation must have $t<s(n)$ tetrahedra. Solving for the equations $n-1 \leq t<s(n)$ for $n=4, \ldots, 9$ gives only the solution $n=9$, $t=8$.

Consider a convex polytope with nine vertices. We show that either no triangulation of size eight is possible or it allows for an invariant triangulation of size eight or less. We can dispose of the case when $P$ has a vertex $v$ of degree three. The reason is such a vertex can be chopped off, the convex hull of the remaining eight points can be triangulated using an invariant triangulation of size no more than $s(8) \leq 7$ by our previous discussion. These at most seven tetrahedra plus the tetrahedron containing $v$ give a size $\leq 8$ invariant triangulation of $P$.

In the graph $G_{\text {face }}$ there could be a vertex with degree more than five, but then our pulling procedure yields an invariant triangulation of at most eight simplices. Hence the only remaining case is that the triangulated planar graph of the triangulated boundary of $P$ has only vertices of degree four or five. If $n_{i}$ denotes the number of vertices of degree $i$, we have that twice the number of edges is $2 \cdot(3 \cdot 9-6)=42=5 n_{5}+4 n_{4}$ and $n_{5}+n_{4}=9$. The only nonnegative integer solution is $n_{5}=6$ and $n_{4}=3$. From these degree restrictions and an easy case analysis, it can be seen that the only triangulated planar graph for the boundary is given in Fig. 6.

We claim that all triangulations that extend this triangulation of the boundary of $P$, will have three or more interior edges and then have nine or more tetrahedra by Lemma 2.1. Assume this is not true. Every boundary triangle is in a tetrahedron. The fourth point of the tetrahedron is in an adjacent triangle, otherwise instantly three interior edges are produced. However, the tetrahedra that cover the triangles $(A, B, X),(A, F, Y)$, and $(B, F, Z)$ will still induce three distinct interior edges.

Remark. Computer experiments showed that often an optimal minimal triangulation of a simplicial polytope is obtained by a pulling triangulation. This has also been ob- 


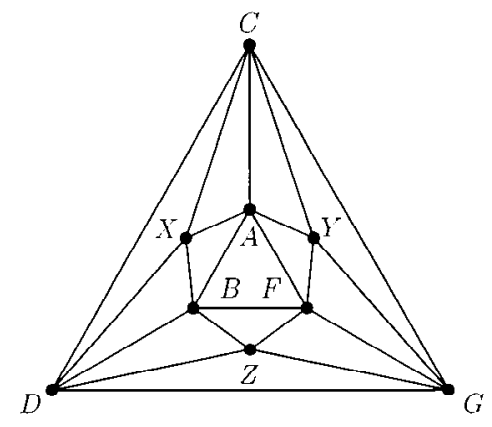

Fig. 6. Unique triangulated planar graph with $n_{5}=6$ and $n_{4}=3$.

served for topological triangulations that extend a triangulated sphere into a triangulated 3-ball (see Section 3.5 of [20]). Our example for Theorem 1.3 (see Fig. 4) shows that this is not always the case.

\section{References}

1. Avis, D., and ElGindy, H. Triangulating point sets in space, Discrete Comput. Geom. 2 (1987), 99111 .

2. Bern, M., and Eppstein, D. Mesh generation and optimal triangulations, in Computing in Euclidean Geometry (D. Z. Du and F. K. Hwang, eds.), World Scientific, Singapore, 1992.

3. Bern, M., Eppstein, D., and Gilbert, J. Provably good mesh generation. J. Comput. System Sci. 48(3) (1994), 384-409.

4. Billera, L., Filliman, P., and Sturmfels, B. Constructions and complexity of secondary polytopes, $A d v$. in Math. 83 (1990), 155-179.

5. Böhm, J. Private communication.

6. Brehm, U. Minimale simpliziale Zerlegungen von 3-Polytopen, Sektion Geometrie, DMV Jahrestagung, Berlin, 1992.

7. Chazelle, B., Edelsbrunner, H., Guibas, L. J., Hershberger, J. E., Seidel, R., and Sharir, M. Selecting heavily covered points, SIAM J. Comput. 23(6) (1994), 1138-1151.

8. Croft, H. T., Falconer, K. J., and Guy, R. K. Unsolved Problems in Geometry, Springer-Verlag, New York, 1991.

9. De Loera, J. A., Hoşten, S., Santos, F., and Sturmfels, B. The polytope of all triangulations of a point configuration, Doc. Math. J. DMV 1 (1996), 103-119.

10. Edelsbrunner, H., Preparata, F. P., and West, D. B. Tetrahedrizing point sets in three dimensions, J. Symbolic Comput. 10 (1990), 335-347.

11. Gritzmann, P., and Klee, V. On the complexity of some basic problems in computational convexity, in Polytopes: Abstract, Convex and Computational (T. Bisztriczky et al., eds.), Kluwer Academic, Dordrecht, 1994, pp. 374-466.

12. Gruber, P. Aspects of approximation of convex bodies, in Handbook of Convex Geometry (P. Gruber and J. Wills, eds.), volume A, North-Holland, Amsterdam, 1993, pp. 319-346.

13. Hughes, R. B., and Anderson, M. R. Simplexity of the cube, Discrete Math. 158(1-3) (1996), 99-150.

14. Lee, C. W. Regular triangulations of convex polytopes, in Applied Geometry and Discrete MathematicsThe Victor Klee Festschrift (P. Gritzmann and B. Sturmfels, eds.), DIMACS Series in Discrete Mathematics and Theoretical Computer Science, volume 4, American Mathematical Society, Providence, RI, 1991, pp. 443-456.

15. Lee, C. W. Subdivisions and triangulations of polytopes, in Handbook of Discrete and Computational Geometry (J. E. Goodman and J. O'Rourke, eds.), CRC Press, New York, 1997, pp. 271-290. 
16. O’Rourke, J. Art Gallery Theorems and Algorithms, Oxford University Press, New York, 1987.

17. Richter-Gebert, J. Finding small triangulations of polytope boundaries is hard, manuscript available at http:/www.inf.ethz.ch/personal/richter.

18. Ruppert, J., and Seidel, R. On the difficulty of triangulating three-dimensional non-convex polyhedra, Discrete Comput. Geom. 7 (1992), 227-253.

19. Schönhardt, E. Über die Zerlegung von Dreieckspolyedern in Tetraeder, Math. Ann. 98 (1928), 309-312.

20. Sleator, D. D., Tarjan, R. E., and Thurston, W. P. Rotation distance, triangulations, and hyperbolic geometry, J. Amer. Math. Soc. 1(3) (1988), 647-681.

21. Ziegler, G. M. Lectures on Polytopes, Springer-Verlag, New York, 1995.

Received August 16, 1999, and in revised form February 29, 2000. Online publication May 9, 2000. 